\title{
Distribution of Random Pulses Forcing a Damped Oscillator Determined in a Finite Time Interval
}

\author{
A. OzGA* \\ AGH University of Science and Technology, Faculty of Mechanic Engineering and Robotics, \\ Department of Mechanics and Vibroacoustics, Al. A. Mickiewicza 30, 30-059 Krakow, Poland
}

\begin{abstract}
Solving a stochastic problem for systems subjected to random series of pulses is, in the present case, aimed at determining of an approximate distribution of amplitudes of random pulses forcing vibrations of an oscillator with damping. The applied model of investigations indicated the source of difficulties connected with interpretation of the obtained results. Another issue discussed in the paper is how a change of the damping coefficient $b$ of the system may result in a decrease of the difference between the actual distribution of random pulses and that determined from the waveform.
\end{abstract}

DOI: 10.12693/APhysPolA.125.A-159

PACS: 45.05.+x, 45.30.+s, 45.90.+

\section{Introduction}

Theoretical and experimental [1] analyses of models of vibrating systems [2] as well as methods used to control such systems [3] are among the topics of research projects [4] carried out in academic centers in Poland [5]. The studies conducted by researchers deal not only with the analysis of vibrations aimed at preventing resonance [6] or degradation of machinery [7], but include also application of numerical analyses [8] of influence of the shapes of piezoelements [9] on their efficiency as vibration reducing actuators [10-11]. The examples listed above refer both to the basic research [8-11] where the teams strive to discover the hitherto unknown interdependencies, and the applied studies oriented at practical applications [1-7].

The considerations presented in this paper dealing with the issue of determining the distribution of stochastic pulses [12] acting on a damped oscillator by way of analysis of a single waveform representing its motion [13], refers to the basic studies having, however, certain application potential. The mathematical model discussed in papers [12-18] allows to compute the distribution of probability for $t \rightarrow \infty$. In technological applications we should take into account a finite time interval which means that the distribution determined from a waveform will be biased with an error. The analyses of causes of errors and minimization of their negative effects have been carried out on the basis of computer simulations.

2. Parameters and description of simulations

Simulation discussed in this paper were carried out for a one-dimensional physical system [12] the state of which is described by means of a single parameter $x$

$$
x(t)=\sum_{0<t_{i}<t} \frac{\eta_{i}}{c} \mathrm{e}^{-b\left(t-t_{i}\right)} \sin \left(\sqrt{a^{2}-b^{2}}\left(t-t_{i}\right)\right),
$$

where the force $f(t)$ exciting vibrations of the system is

*corresponding author; e-mail: aozga@agh.edu.pl defined as a series of random pulses with amplitudes $\eta_{i}$ occurring at random instants of time $t_{i}$ :

$$
f(t)=\sum_{t_{i}<t} \eta_{i} \delta\left(t-t_{i}\right) .
$$

The intervals between the pulses and amplitudes of the pulses are independent random variables [13]. Depending on the context, three distributions $\Phi_{i}$ of the pseudorandom variable $\eta_{i}$ are considered in this paper:

$$
\begin{aligned}
& \text { 1. } \Phi_{1}: p\left(\eta_{1}\right)=0, p\left(\eta_{2}\right)=1, p\left(\eta_{3}\right)=0 \\
& \text { 2. } \Phi_{2}: p\left(\eta_{1}\right)=0.33, p\left(\eta_{2}\right)=0.33, p\left(\eta_{3}\right)=0.34 \\
& \text { 3. } \Phi_{3}: p\left(\eta_{1}\right)=0.4767, p\left(\eta_{2}\right)=0, p\left(\eta_{3}\right)=0.5233 .
\end{aligned}
$$

Another assumption made in the model consists in that distributions of random variables which are time intervals $\left(t_{i}-t_{i-1}\right)$ between podsequent pulses have the same exponential distribution $F(x)$ and these variables are stochastically independent, namely

$$
F(\tau)= \begin{cases}1-\exp (-\lambda \tau) & \text { if } \tau \geq 0 \\ 0 & \text { if } \tau<0\end{cases}
$$

where the constant $\lambda$ is the pulse occurrence frequency. Taking into account one-dimensional systems [14], recording the time of occurrence and amplitude of the pulsed action, and neglecting the place of the pulse, we are able to explain the causes of the problems connected with interpretation of the obtained results for different pulse occurrence frequencies $\lambda[15]$ equal to 10 , $10^{2}, 10^{3}$, and $10^{4} \mathrm{~s}^{-1}$.

In order to execute a simulation for a single realization, it is necessary to sample podsequent values of $\eta_{i}$ as well as podsequent time intervals $\left(t_{i}-t_{i-1}\right)$ between the pulses [16] for a selected or given distribution $\Phi_{i}$ and in accordance with a selected or given pulse occurrence frequency $\lambda$. Then, putting the sampled values in (1), we calculate $x(t)$ which allows to determine stochastic moments $m_{n}(t)$ and the distribution of pulses $\tilde{p}_{i}(t)$ which was described in detail in papers $[12-14,17]$. 
Simulation studies regarding the oscillator were executed for RLC systems [15]. The parameters $a=$ 300179.47 and $b=8280.97$ and $\eta$ assumed three amplitude values, $\eta_{1}=845,778.47, \eta_{2}=\eta_{1} / 2$, and $\eta_{3}=\eta_{1} / 10$ were approximated from the response of the system to a single pulse [16]. The methods of approximation are presented in $[18]^{\dagger}$. Simulations as well as calculations were carried out in MATLAB environment [19].

\section{Methods of investigations}

Let $\tilde{p}_{i}(t)$ denote the distribution which is recorded from the waveform, and $p_{i}$ - the distribution imposed in simulation, while the index $i$ denotes reference to the pulse of a given amplitude - e.g., $p_{1}(\mathrm{t})$ is calculated for the pulse $\eta_{1}$.

The differences $\tilde{p}_{i}(t)-p_{i}$ are too strongly influenced by the properties of the generator of pseudorandom numbers with uniform distribution used in the investigations. In MATLAB environment, in which the investigations were conducted, achieving probability with the assumed precision of $10^{-4}$ is possible only for $10^{9}$ elements in a statistic sample. For low pulse occurrence frequency values, it would require too long time interval to be analyzed.

Hence, in the study we look for the most important causes of the occurrence of deviations $\tilde{p}_{i}(t)-\bar{p}_{i}(t)$, where $\bar{p}_{i}(t)$ is the distribution executed by the generator of pseudorandom numbers - calculated separately for each of the generated realizations.

\subsection{The results of analysis related to pulse occurrence frequency}

Neglecting the details regarding the changes that occur depending on amplitude of a pulse, examining the distribution $\Phi_{1}$ including only the pulses of the amplitude $\eta_{2}$ we can analyze the difficulties connected with interpretation of the obtained results depending on the pulse occurrence frequency.

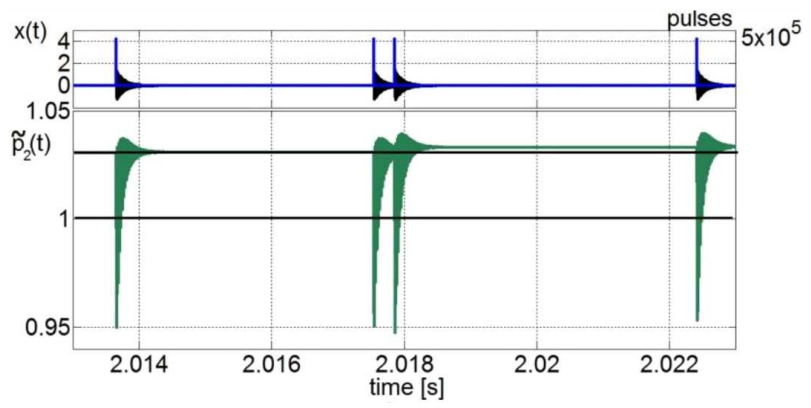

Fig. 1. The waveform, pulses, and estimators $\tilde{p}_{2}$ corresponding to $\Phi_{1}, \lambda=10^{2} \mathrm{~s}^{-1}$.

†The above-mentioned parameters applied in the simulations discussed in this paper are connected with the system consisting of inductivity $L=5 \mathrm{mH}$, capacity $C=2 \mathrm{nF}$, and a voltage source. All the elements were connected in series, and the resistance $\mathrm{R}$ came from the physical components making up the circuit.
The first issue that deserves attention is the fact that while analyzing a single waveform, one should not determine the distributions of pulses at certain constant points of time, but at certain time intervals with breaks between them, because at the moment when a pulse occurs, the difference $\tilde{p}_{i}(t)-\bar{p}_{i}(t)$ increases (Fig. 1). Additionally, if two pulses occur in such a short period of time that the vibrations will not disappear, the changes that occur in the system increase the differences $\tilde{p}_{i}(t)-\bar{p}_{i}(t)$ (Fig. 2).

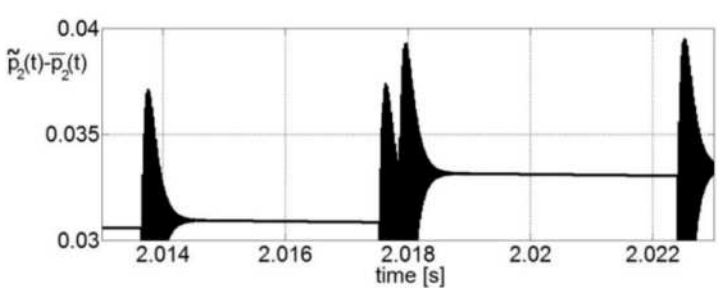

Fig. 2. The difference $\tilde{p}_{2}(t)-\bar{p}_{2}(t)$ for the vibrations presented in Fig. 1.

The idea of constructing a mathematical model consisted in recording a single waveform, but it is necessary to execute statistical analysis to recognize how long the time interval should be in order to reduce the error issuing from the occurrence of another pulse at a definite time-point to minimum. Therefore, 100 independent waveforms were generated for each of the three distributions $\Phi_{i}$ and for $\lambda=10,10^{2}, 10^{3}$, and $10^{4} \mathrm{~s}^{-1}$ which gives the total of 12 analyzed cases. The obtained differences $\tilde{p}_{i}(t)-\bar{p}_{i}(t)$ were grouped into histograms which, with the passage of time come close to the Gaussian distribution, indicated by the following indices of descriptive statistics: mean value, median, standard deviation, kurtosis, and skewness. The indices characterize the collected data in the aspect of the most probable value, the spread of this value, and the shape of the histogram, indicating, at the same time, the difficulty at interpreting the obtained results. These histograms for $t=300,600$, and $900 \mathrm{~s}$ are presented in the following figures.

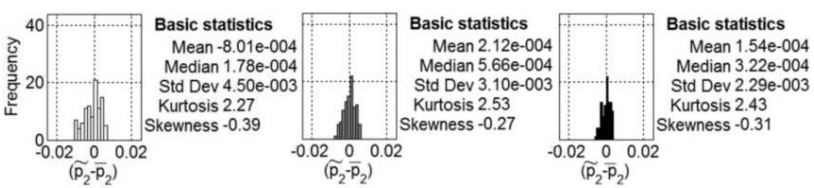

Fig. 3. Histograms $\tilde{p}_{2}(t)-\bar{p}_{2}(t)$ corresponding to $\Phi_{1}$ and $\lambda=10 \mathrm{~s}^{-1}$.

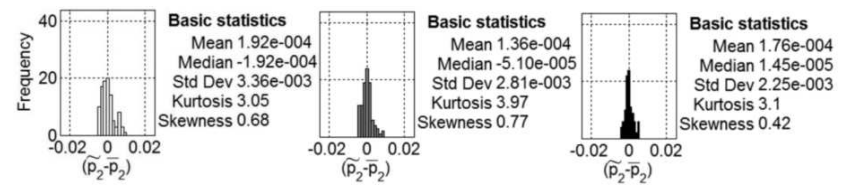

Fig. 4. Histograms $\tilde{p}_{2}(t)-\bar{p}_{2}(t)$ corresponding to $\Phi_{1}$ and $\lambda=10^{2} \mathrm{~s}^{-1}$. 


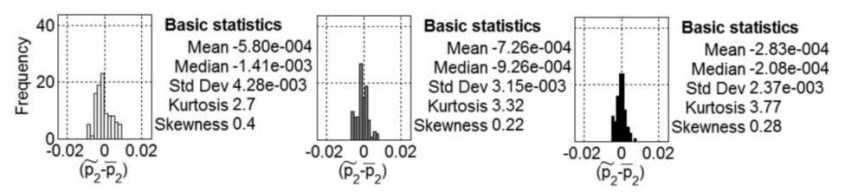

Fig. 5. Histograms $\tilde{p}_{2}(t)-\bar{p}_{2}(t)$ corresponding to $\Phi_{1}$ and $\lambda=10^{3} \mathrm{~s}^{-1}$.

For low pulse occurrence frequency values, the difficulties described above occur during the whole analyzed time interval, hence the standard deviation of $\tilde{p}_{2}(t)-\bar{p}_{2}(t)$ determined in the statistical studies for 100 independent waveforms for $\lambda=10 \mathrm{~s}^{-1}$ (Fig. 3, Fig. 6) is higher than the standard deviation determined for the pulse occurrence frequency $\lambda=10^{2} \mathrm{~s}^{-1}$ (Fig. 4, Fig. 6), but lower than the standard deviation determined for $\lambda=10^{4} \mathrm{~s}^{-1}$ (Fig. 5, Fig. 6).

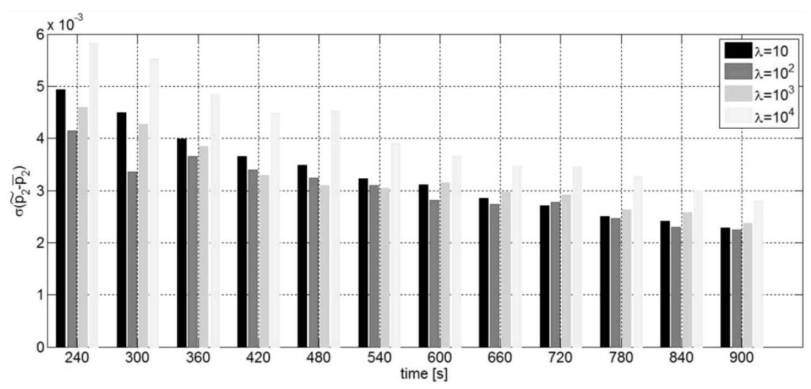

Fig. 6. The standard deviation of $\tilde{p}_{2}(t)-\bar{p}_{2}(t)$ determined in statistical investigations for 100 independent waveforms for $\lambda=10,10^{2}, 10^{3}, 10^{4} \mathrm{~s}^{-1}$ corresponding to $\Phi_{1}$.

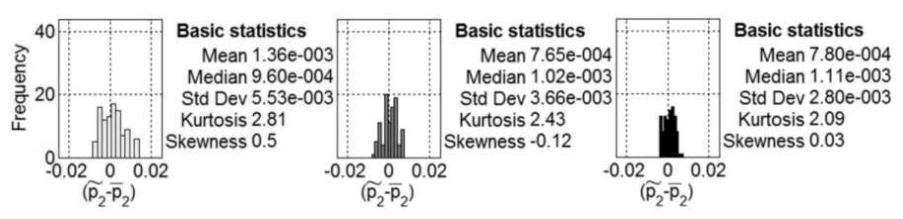

Fig. 7. Histograms $\tilde{p}_{2}(t)-\bar{p}_{2}(t)$ corresponding to $\Phi_{1}$ and $\lambda=10^{4} \mathrm{~s}^{-1}$.

Further difficulties in interpretation of the obtained results can be studied while comparing the data presented in diagram 7 :

- For $\lambda=10^{2} \mathrm{~s}^{-1}$, the standard deviation of $\tilde{p}_{2}(t)-$ $\bar{p}_{2}(t)$ does not always decrease with the passage of time, e.g. it is higher for $t=360 \mathrm{~s}$ than for $t=$ $300 \mathrm{~s}$;

- For $t=300 \mathrm{~s}$, the standard deviation of $\tilde{p}_{2}(t)-\bar{p}_{2}(t)$ for $\lambda=10 \mathrm{~s}^{-1}$ is higher than the standard deviation computed for $\lambda=10^{2} \mathrm{~s}^{-1}$ and for $\lambda=10^{3} \mathrm{~s}^{-1}$, while for $t=720 \mathrm{~s}$, the trend is opposite.

In turn, for high occurrence frequencies $\lambda$ on the basis of an analysis of a single waveform (Fig. 8) it can be

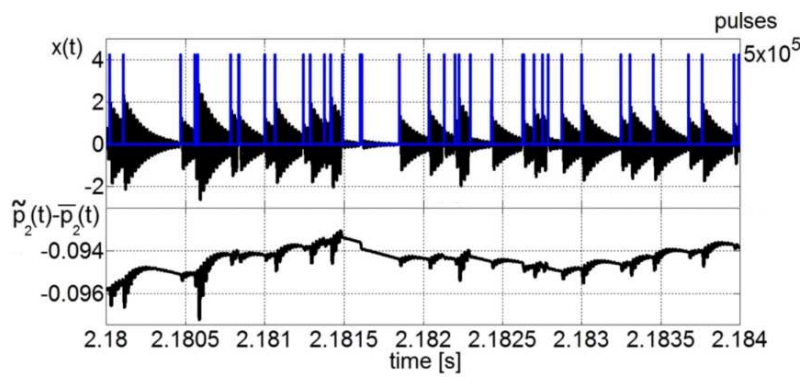

Fig. 8. The waveform, pulses and difference $\tilde{p}_{2}(t)-$ $\bar{p}_{2}(t)$ corresponding to $\Phi_{1}, \lambda=10^{4} \mathrm{~s}^{-1}$.

seen that the influence of a single pulse is lower, but the absolute value of the difference $\tilde{p}_{i}(t)-\bar{p}_{i}(t)$ is higher than that for low pulse occurrence frequencies. Hence the standard deviation of $\tilde{p}_{2}(t)-\bar{p}_{2}(t)$ determined in statistical analyses for 100 independent waveforms $\lambda=10^{4} \mathrm{~s}^{-1}$ (Fig. 5, Fig. 6) is higher than the standard deviation of $\tilde{p}_{2}(t)-\bar{p}_{2}(t)$ determined for the frequency $\lambda=10^{3} \mathrm{~s}^{-1}$ (Fig. 7, Fig. 6).

It is worth noticing that for high pulse occurrence frequencies $\lambda$ it often happens that the podsequent pulses damp the vibrations of the system evoked by previous pulses (Fig. 8, $t=2.1815 \mathrm{~s}$ ). Hence, the higher the pulse frequency, the slower the standard deviation of $\tilde{p}_{2}(t)-\bar{p}_{2}(t)$ decreases (Fig. 6).

3.2. The results of analysis related to pulse amplitudes

The changes that occur depending on the amplitude of a pulse may be studied while examining the distribution $\Phi_{2}$ analyzing a single waveform and carrying out statistical analysis. Figure 9 allows to draw conclusions independently of the pulse occurrence frequency.

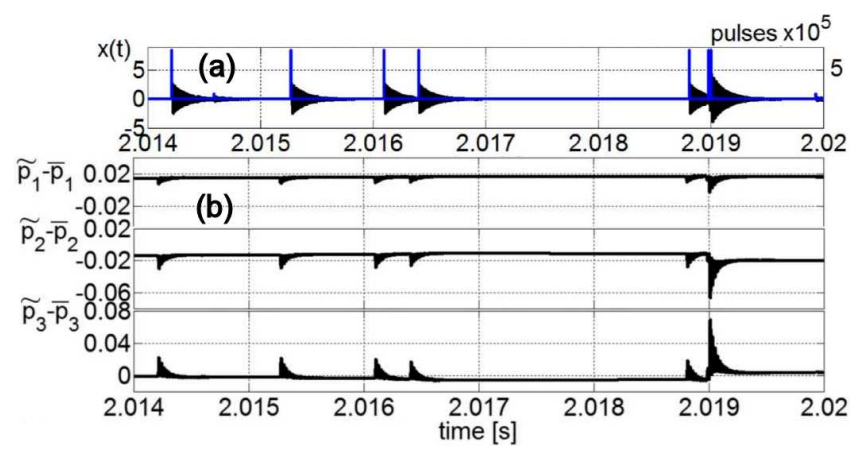

Fig. 9. (a) The waveform and pulses corresponding to $\Phi_{2}, \lambda=10^{3} \mathrm{~s}^{-1},(\mathrm{~b})$ The difference $\tilde{p}_{i}(t)-\bar{p}_{i}(t)$ corresponding to vibration presented in part (a).

- The strongest pulses are the cause of the largest difference $\tilde{p}_{i}(t)-\bar{p}_{i}(t)$.

- Independently of the amplitude of the pulse $\lambda_{i}$, the difference $\tilde{p}_{3}(t)-\bar{p}_{3}(t)$ is the highest while $\tilde{p}_{1}(t)-\bar{p}_{1}(t)$ is the lowest, which is confirmed by the histograms (Figs. 10, 11). 


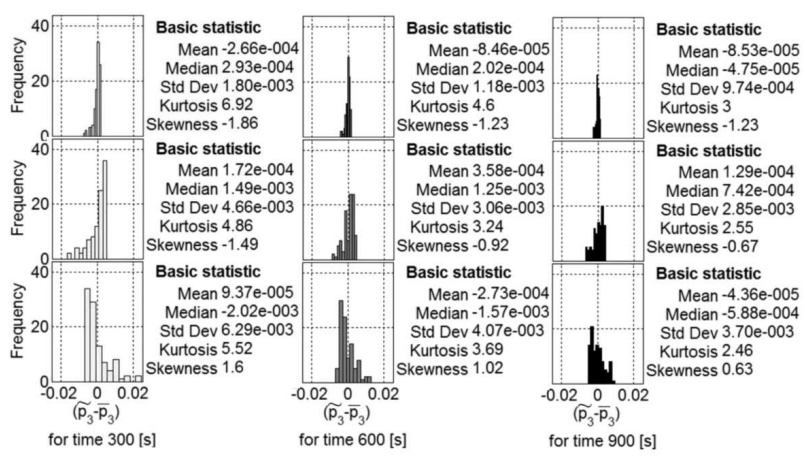

Fig. 10. Histograms $\tilde{p}_{i}(t)-\bar{p}_{i}(t)$ corresponding to $\Phi_{2}$ and $\lambda=10 \mathrm{~s}^{-1}$.

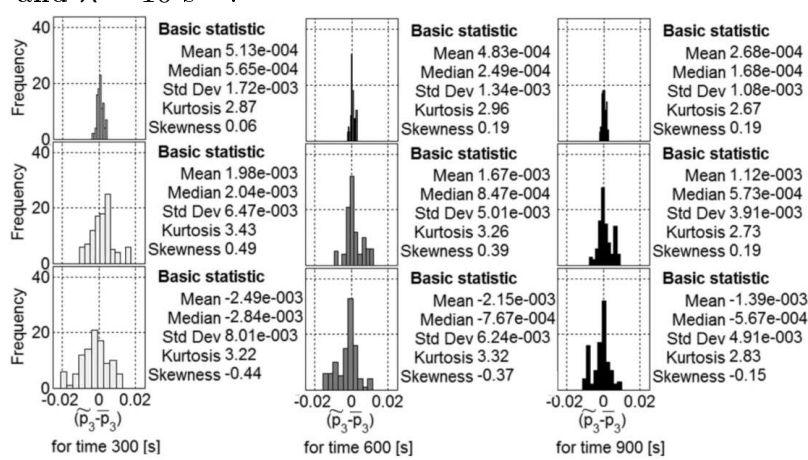

Fig. 11. Histograms $\tilde{p}_{i}(t)-\bar{p}_{i}(t)$ corresponding to $\Phi_{2}$ and $\lambda=10^{4} \mathrm{~s}^{-1}$.

\subsection{The greatest difficulty connected with interpretation of the obtained results}

Comparing the standard deviation of $\tilde{p}_{i}(t)-\bar{p}_{i}(t)$ determined for 100 independent waveforms and three distributions $\Phi_{1}, \Phi_{2}, \Phi_{3}$, contradictory results are obtained, which constitutes the greatest difficulty connected with interpretation of the obtained results (Fig. 12).

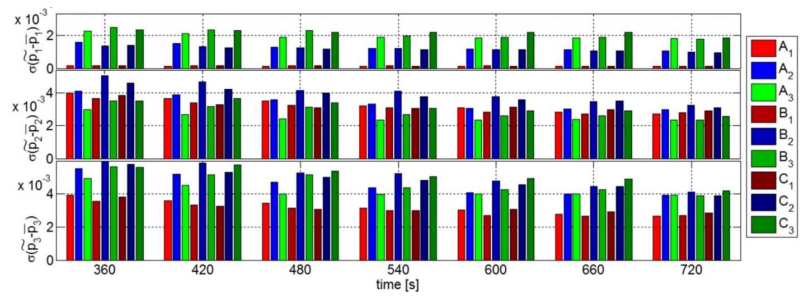

Fig. 12. The standard deviation of $\tilde{p}_{i}(t)-\bar{p}_{i}(t)$ corresponding for $A_{1}, B_{1}$ and $C_{1}$ to $\lambda=10 \mathrm{~s}^{-1}$; for $A_{2}$, $B_{2}$ and $C_{2}$ to $\lambda=10^{2} \mathrm{~s}^{-1}$; for $A_{3}, B_{3}$ and $C_{3}$ to $\lambda=10^{2} \mathrm{~s}^{-1}$, where the letter $A$ denotes distributions $\Phi_{1}, B$ - distributions $\Phi_{2}$ and $C$ - distributions $\Phi_{3}$.

It is hard to find the interrelations $\sigma\left(\tilde{p}_{i}(t)-\bar{p}_{i}(t)\right)$ and the pulses of the given amplitude $\eta_{i}$ depending on the distribution, for instance:

1. for $\sigma\left(\tilde{p}_{1}(t)-\bar{p}_{1}(t)\right)$, the highest bars are marking the distribution $\Phi_{3}$ - the distribution in which the largest number of pulses $\eta_{1}$ occurs;
2. for $\sigma\left(\tilde{p}_{2}(t)-\bar{p}_{2}(t)\right)$, the tallest bars correspond to the distribution $\Phi_{2}$ - the distribution in which the largest number of pulses $\eta_{2}$ occurs, while

3. $\sigma\left(\tilde{p}_{3}(t)-\bar{p}_{3}(t)\right)$ varies depending on time and pulse occurrence frequency.

The above-discussed interdependence between the standard deviation of $\tilde{p}_{i}(t)-\bar{p}_{i}(t)$ for different $\lambda$ does not allow to find interrelations (Fig. 12), e.g., for $t=540 \mathrm{~s}$ and for the difference $\tilde{p}_{3}(t)-\bar{p}_{3}(t)$, the maximum $\sigma$ was determined for $\lambda=10^{2} \mathrm{~s}^{-1}$ compared to $\lambda=10^{3} \mathrm{~s}^{-1}$ for $t=660 \mathrm{~s}$

Similarly, there seems to be no dependence between the standard deviation of $\tilde{p}_{i}(t)-\bar{p}_{i}(t)$ for different distributions, e.g., for $t=420 \mathrm{~s}$, for both $\lambda=10$ and $\lambda=10^{2} \mathrm{~s}^{-1}$ the highest bars represent the distribution $\Phi_{2}$, while for $\lambda=10^{3} \mathrm{~s}^{-1}$ the highest bar corresponds to $\Phi_{3}$.

In view of the above, it is impossible to assess how long the waveform taken into consideration should be in order to prevent the difference $\tilde{p}_{1}(t)-\bar{p}_{1}(t)$ from increasing dramatically when the podsequent pulses occur.

And yet the scientific research shows that there are two possible ways of interfering with the algorithm determining the distributions of pulses. If we supplement the algorithm computing $\tilde{p}_{i}(t)$ with a condition that the measuring system should delay calculation of distributions until the absolute value of deviation $x(t)$ of the oscillator is low, e.g., lower than 0.001 , we shall obtain results that eliminate the largest differences $\tilde{p}_{i}(t)-\bar{p}_{i}(t)$. For low frequency of occurrence of the hits, introduction of this additional condition will be more significant than for high pulse occurrence frequencies. The other possible way of interfering will be discussed in the next paragraph.

\section{Decreasing the difference between the actual distribution of pulses and that determined from waveforms through increasing the damping coefficient $b$}

In order to check in what way a change of parameters of the vibrating system influences the precision of the calculated distributions, in this section we change the damping coefficient $b$ by increasing it ten times up to the value $82,809.7$. Using the files generated for the distribution $\Phi_{2}$ including the times of action $t_{i}$ and pulse amplitudes $\eta_{i}$, we will compute $\tilde{p}_{i}(t)-\bar{p}_{i}(t)$ once more. The remaining parameters of the simulation: coefficient $a$, as well as pulse amplitudes $\eta_{1}, \eta_{2}$, and $\eta_{3}$ remain unchanged. The results of the simulations are presented in the histograms below.

The indices of descriptive statistics calculated in this section: mean value, median, standard deviation, kurtosis, and skewness indicate that the examined differences $\tilde{p}_{i}(t)-\bar{p}_{i}(t)$ are better for the systems with strong damping that for those with weak damping (compare Fig. 10 with Fig. 13 and Fig. 11 with Fig. 14). The histograms show the results for the lowest and the highest of the considered pulse frequencies $\lambda$; the remaining cases for 


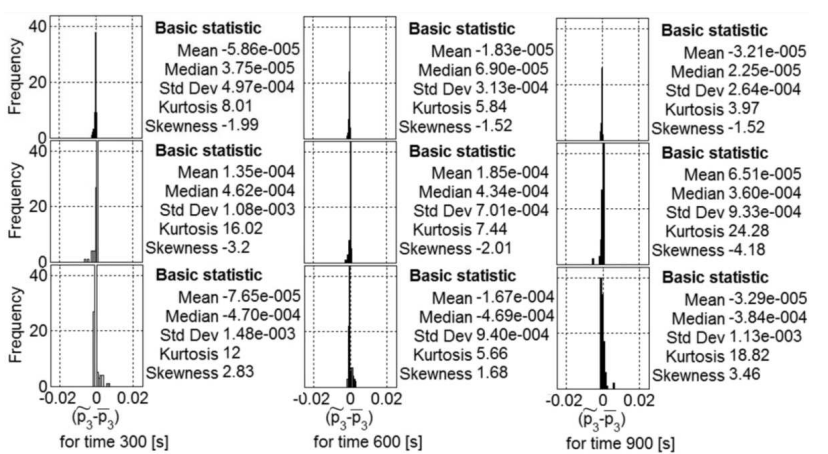

Fig. 13. Histograms $\tilde{p}_{i}(t)-\bar{p}_{i}(t)$ corresponding to $\Phi_{2}$ and $\lambda=10 \mathrm{~s}^{-1} .100$ distributions forced by the same pulses at the same time as the distributions presented in Fig. 10, but for the damping coefficient of the vibrating system, $b=82,809.7$, increased 10 times.

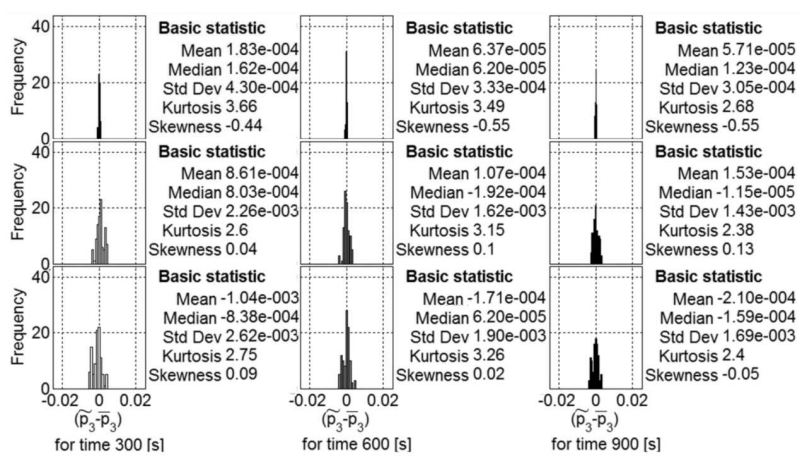

Fig. 14. Histograms $\tilde{p}_{i}(t)-\bar{p}_{i}(t)$ corresponding to $\Phi_{2}$ and $\lambda=10^{4} \mathrm{~s}^{-1} .100$ distributions forced by the same pulses at the same time as the distributions presented in Fig. 10, but for the damping coefficient of the vibrating system, $b=82,809.7$, increased 10 times.

different pulse occurrence frequencies (Fig. 15) and different distributions confirm the above conclusions.

Unfortunately, analysis of the data included in Fig. 15 will not lead to answering the question in what way the pulse occurrence frequency should be related to the length of the time interval necessary Again, certain inconsistencies are found here, for example when $t=720 \mathrm{~s}$, for determining the distributions. $\sigma\left(\tilde{p}_{2}(t)-\bar{p}_{2}(t)\right)$ for

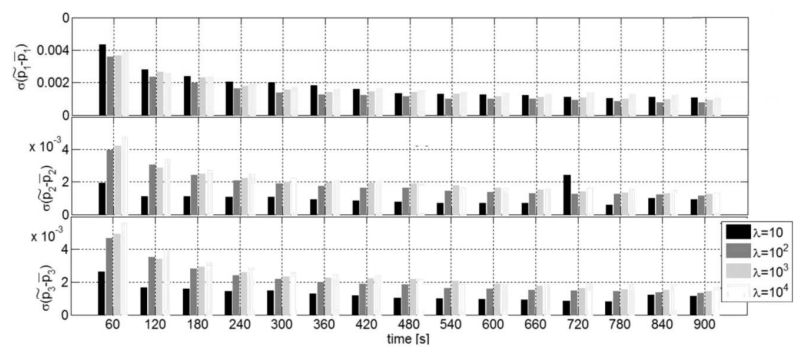

Fig. 15. The standard deviation of $\tilde{p}_{i}(t)-\bar{p}_{i}(t)$ determines in statistical investigations for 100 independent waveforms for $\lambda=10,10^{2}, 10^{3}, 10^{4} \mathrm{~s}^{-1}$ corresponding to $\Phi_{2}$. $\lambda=10 \mathrm{~s}^{-1}$ is the highest, but when $t=840 \mathrm{~s}$, it is the lowest. The change of the parameters of a vibrating system will not change the difficulties issuing from the analysis of the systems subjected to random series of pulses; it will merely decrease the difference between the distribution recorded from the waveform and that which actually has forced this waveform.

\section{Summary}

The applied model of investigations has pointed out the source of difficulties connected with interpretation of the obtained results. The decrease of the difference between the distribution of hits that forced vibrations of the system and the distributions determined from a single waveform is possible by way of increasing the damping coefficient.

\section{References}

[1] M. Sibielak, Mechanical Systems and Signal Processing 36, 118 (2013).

[2] W. Raczka, M. Sibielak, and H. Bah, Proc. 15th Int. DAAAM Symposium: intelligent manufacturing E automation: globalisation - technology - men nature, Ed.: B. Katalinic, DAAAM International, Vienna 2004

[3] J. Konieczny, J. Kowal, W. Raczka, M. Sibielak, J. Low Frequency Noise, Vibration, Active Control 32, 81 (2013).

[4] M. Sibielak, J. Konieczny, J. Kowal, W. Raczka, D. Marszalik, J. Low Frequency Noise, Vibration, Active Control 32, 99 (2013).

[5] W. Raczka, M. Sibielak, J. Kowal, J. Konieczny, J. Low Frequency Noise, Vibration, Active Control 32, 117 (2013).

[6] E. Rusiński, P. Moczko, D. Pietrusiak, G. Przybyłek, Journal of Mechanical Engineering 59, 556 (2013).

[7] J. Czmochowski, P. Moczko, P. Odyjas, D. Pietrusiak, Maintenance and Reliability 16, 305 (2014).

[8] M. Kozupa, J. Wiciak, Acta Phys. Pol. A 118, 95 (2010).

[9] A. Brański, Acoustic Waves, ch. 18, InTech, Rijeka, Chorwacja 2011, pp. 397.

[10] M. S. Kozień, Acta Phys. Pol. 123, 1029 (2013).

[11] M. Wiciak, Acta Phys. Pol. A 121, A-142 (2012).

[12] M. Jabłoński, A. Ozga, Acta Phys. Pol. 118, 174 (2010).

[13] M. Jabłoński, A. Ozga, Mechanics 29, 163 (2010).

[14] M. Jabłoński, A. Ozga, T. Korbiel, P. Pawlik, Acta Phys. Pol. 119, 997 (2011).

[15] M. Jabłoński, A. Ozga, Acta Phys. Pol. 121, A-174 (2012).

[16] M. Jabłoński, A. Ozga, Arch. Acoust. 34, 601 (2009).

[17] M. Jabłoński, A. Ozga Distribution of random pulses acting on a vibrating system as a function of its motion, AGH University of Science and Technology Press, Kraków 2013.

[18] A. Ozga, Acta Phys. Pol. 123, 1034 (2013).

[19] W. Regel, Mathematical Statistics in MATLAB, MIKOM, Warszawa 2004, (in Polish). 